Cahiers philosophiques, « Mesurer », $\mathrm{n}^{\circ} 135,4^{\mathrm{e}}$ trimestre 2013, p. 36-50.

\title{
Mesure relative et mesure absolue
}

\author{
Olivier REY ${ }^{1}$
}

RÉSUMÉ. - Dans l'espace homogène que la science moderne a substitué à l'ancien cosmos, au sein d'un espace infiniment étendu et infiniment divisible, sans éléments minimaux ou maximaux propres à fixer une échelle ni rien qui puisse faire borne ou limite, la mesure semblait vouée à n'être qu'une notion purement relative. Pourtant, dans ses derniers travaux, Galilée a montré que la variation non linéaire de certaines variables physiques par rapport à d'autres suffit à donner une valeur absolue à certains ordres de grandeur. L'échelle des phénomènes n'est pas un paramètre qui vient s'ajouter à leur forme, elle influe de manière déterminante sur cette forme qui, en retour, ne peut exister qu'à certaines échelles.

\section{Mesurer dans un cosmos ou dans un espace géométrique infini}

Le passage du système de Ptolémée au système de Copernic peut, une fois effectué, être envisagé comme un passage du géocentrisme à l'héliocentrisme et, à cet égard, sembler se résumer essentiellement à un changement d'origine dans un référentiel physique, assorti de quelques révisions astronomiques - les planètes autres que la Terre ne tournent pas autour de celle-ci mais autour du Soleil. De ce point de vue, les oppositions passionnées qu'a suscitées ce changement de système paraissent disproportionnées, voire insensées. Freud y a décelé les symptômes d'une blessure narcissique : l'être humain ne supportait pas de ne plus être au centre du monde ${ }^{2}$. Aussi clairvoyant que Freud ait pu souvent se montrer, il s'est ici trompé du tout au tout. Aux temps archaïques où la Terre était conçue comme plate, elle était sous le ciel. Quand on se l'est représentée ronde, la seule façon pour qu'elle demeurât partout sous les cieux était qu'elle fût au centre, entourée par eux. Ainsi la place qu'elle occupait dans le schéma ptolémaïque était-elle tout sauf un lieu privilégié : c'était au contraire l'endroit le pire du cosmos - endroit qu'il lui revenait d'occuper en raison de sa dignité moindre par rapport aux astres. Aussi les adversaires les plus farouches du système de Copernic n'étaient-ils pas

\footnotetext{
${ }^{1}$ Institut d'histoire et de philosophie des sciences et des techniques (CNRS - Université Paris 1).

${ }^{2}$ Voir la dix-huitième leçon d'introduction à la psychanalyse, « La fixation au trauma ; l'inconscient », professée en 1916, et Une difficulté de la psychanalyse [1917].
} 
mus par la vanité blessée de ne plus être au centre : ils étaient au contraire outrés par l'orgueil insensé consistant à mettre la Terre au même rang que les astres. Montaigne, qu'il n'est pas d'usage de considérer comme un esprit borné ou rétrograde, est animé par ce sentiment lorsqu'il écrit dans les Essais : «La plus calamiteuse et frêle de toutes les créatures, c'est l'homme, et quant et quant [i.e. en même temps] la plus orgueilleuse. Elle se sent et se voit logée ici, parmi la bourbe et la fiente du monde, attachée et clouée à la pire, plus morte et croupie partie de l'univers, au dernier étage du logis et le plus éloigné de la voûte céleste, avec les animaux de la pire condition des trois [i.e. terrestre, aquatique et aérienne]; et se va plantant par imagination au-dessus du cercle de la lune et ramenant le ciel sous ses pieds ${ }^{3}$. » $\mathrm{Au}$ demeurant, ceux qui imaginent que l'héliocentrisme a porté atteinte au narcissisme humain auraient dû s'aviser d'une difficulté : l'enfer était tenu pour être situé sous terre. Autrement dit, à l'extrême centre du monde était son lieu, non le plus noble, mais le plus abject.

Mais pour quelle raison, alors, s'opposer avec tant de véhémence à une théorie qui rehaussait l'homme? L'humilité n'est pas la seule explication. Certes, dans l'ancienne distribution cosmique, l'homme avait un séjour peu reluisant; mais, du moins, avait-il une place à lui. Dans le nouveau monde, il n'a plus aucune place assignée. Montaigne parle de la vanité dont l'homme fait preuve en se situant parmi les étoiles : c'est le signe que, pour sa part, il se situe toujours dans l'ancien cosmos, car dans le nouvel univers la vanité disparaît, les étoiles n'ayant plus rien de noble. On avait, auparavant, une Terre placée plus bas que tout - mais qui, précisément, situait l'homme dans un espace ordonné et signifiant. La Terre est désormais une planète parmi d'autres, perdue dans l'immensité, dans «le silence éternel des espaces infinis » qui effraie le libertin de Pascal. En effet, à partir du moment où la distinction entre le monde terrestre (sublunaire) et le monde céleste s'efface, les lieux deviennent équivalents, l'espace s'homogénéise, se confond avec un espace géométrique euclidien sans lieux privilégiés, isotrope, infini.

La question de la mesure s'en trouve bouleversée. Dans un cosmos, c'est-à-dire, au sens premier du terme, un ensemble bien ajusté, harmonieux, et fini, les dimensions d'un objet font partie de son être et participent à son ajointement au tout. Certes, les mesures auxquelles on procède sont toujours, nécessairement, relatives : on compare la taille d'un objet à celle d'un autre, éventuellement pris comme référence ou unité de mesure. Il n'en demeure pas moins que la taille de chaque entité, indépendamment de l'unité dans laquelle on l'évalue, a également, de par l'insertion de cette entité dans le cosmos, un sens absolu. Dans l'espace géométrique euclidien qui, avec l'avènement de la science moderne, va devenir le cadre

\footnotetext{
${ }^{3}$ Essais [1580-1588], livre II, chapitre XII : «Apologie de Raimond Sebond », in CEuvres complètes, Paris, Seuil, 1967, p. 187. Plus généralement, sur le statut de la Terre dans le système de Ptolémée, voir Rémi Brague, «Geocentrism as a Humiliation for Man », Medieval Encounters n 3, 1997, p. 187-210.
} 
explicite ou implicite pour penser l'univers, il n'en est pas ainsi. Cet espace est à la fois infiniment étendu et, le point étant sans dimension, infiniment divisible. Il n'y a donc pas plus d'élément maximal que d'éléments minimaux susceptibles de fixer une échelle; rien non plus qui, dans l'homogénéité géométrique, puisse faire borne, limite. Pascal fut l'un des premiers, au XVII ${ }^{\mathrm{e}}$ siècle, à prendre pleinement conscience de la nouvelle situation et à s'appliquer à en tirer les conséquences. Tel est le propos du fragment le plus développé de toutes les Pensées et, comme en témoignent les multiples corrections dont il a été l'objet, l'un des plus travaillés, intitulé «Disproportion de l'homme ${ }^{4}$. Par disproportion, il ne faut pas entendre ici « mauvaise proportion », ou « taille excessive », comme le voudrait l'usage courant du terme, mais absence de référence par rapport à laquelle on pourrait «proportionner ». "Voilà où nous mènent les connaissances naturelles », écrit Pascal en commençant - confirmant par ces mots que c'est bien la nouvelle science qui le conduit à développer les considérations qui suivent. L'homme se retrouve sans mesure absolue, sans proportion, car « rien ne peut fixer le fini entre les deux infinis qui l'enferment et le fuient »: le fini est tout aussi bien néant à l'égard de l'infini que tout à l'égard du néant. Pour mieux faire comprendre ce dernier point, Pascal présente à l'homme un ciron, ce minuscule acarien qui, à son époque, était le plus petit animal connu. De son interlocuteur, il réclame qu'il pense aux jambes et aux jointures dont cet animal, tout juste visible à l'œil nu, est pourvu, puis aux veines qui les irriguent, au sang qui y circule, aux humeurs dans ce sang, aux gouttes dans ces humeurs, aux vapeurs dans ces gouttes, jusqu'au dernier objet que l'imagination, poursuivant ses divisions, parvienne à concevoir. Puis l'homme, qui croyait enfin avoir atteint «l'extrême petitesse de la nature », est invité à découvrir dans ce plus infime des objets un abîme nouveau : «qu'il y voie une infinité d'univers, dont chacun a son firmament, ses planètes, sa terre, en la même proportion que le monde visible; dans cette terre, des animaux, et enfin des cirons, dans lesquels il retrouvera ce que les premiers ont donné, et trouvant encore dans les autres la même chose sans fin et sans repos, qu'il se perde dans ces merveilles aussi étonnantes dans leur petitesse que les autres par leur étendue ; car qui n'admirera que notre corps, qui tantôt n'était pas perceptible dans l'univers, imperceptible lui-même dans le sein du tout, soit à présent un colosse, un monde ou plutôt un tout à l'égard du néant où l'on ne peut arriver ? »

On ne saurait mieux exprimer la relativité de toute mesure qui s'impose à la pensée moderne. Si Pascal se distingue, ici, c'est surtout par ses qualités littéraires : l'idée qu'il expose est, quant à elle, partagée par la plupart des esprits éclairés de son temps.

\section{La leçon de Galilée}

Il se trouve cependant que l'expérience de pensée qu'il propose, et qui veut que les mêmes phénomènes se retrouvent d'échelle en échelle, à l'infini, repose sur un postulat faux.

\footnotetext{
${ }^{4}$ Pensées [1669], fragment 185 de l'édition Le Guern, Paris, Gallimard, 1977 (Br. 161, Laf. 174).
} 
Elle est inspirée par le nouveau regard scientifique sur le monde; et, pourtant, ce même regard l'invalide. On pourrait en appeler, pour le montrer, à la science contemporaine. Selon les théories actuellement en cours, en effet, il se pourrait que l'univers soit d'extension finie (mais de taille croissante, et sans frontières, comme la notion mathématique de variété permet de le concevoir), il n'est en tout cas ni homogène ni isotrope (c'est-à-dire qu'il ne présente pas les mêmes caractéristiques en tout point et en toute direction, car la métrique de l'espacetemps est modifiée par la matière et l'énergie, conformément aux équations d'Einstein). Par ailleurs, on sait que les forces prépondérantes ne sont pas les mêmes selon l'échelle, ce qui donne lieu à des phénomènes de natures elles-mêmes très différentes - le monde subatomique ne ressemble nullement au monde macroscopique. Quant à la division à l'infini de l'espace, elle est probablement dépourvue de sens physique (selon la théorie des supercordes, par exemple, il n'y aurait pas de sens à parler d'une longueur inférieure à la longueur de Planck, de l'ordre de $10^{-35} \mathrm{~m}$ ). Cela étant, il n'est nullement besoin de se référer à des théories aussi récentes et aussi sophistiquées, que Pascal et son siècle ne pouvaient qu'ignorer, pour contredire son argument. À cette fin, la physique de Galilée suffit.

La chose, a priori, peut paraître étonnante. Galilée, en effet, a été un des grands artisans de la liquidation de l'ancien cosmos, fini et hiérarchisé, au profit d'un univers infini écrit, de part en part, en langue mathématique ${ }^{5}$. Tycho Brahe en 1572, Kepler en 1604, avaient noté et décrit l'apparition de nouvelles étoiles dans le ciel, qui ne se révélait donc pas aussi immuable qu'on l'avait cru (l'apparition de ces étoiles, qualifiées à l'époque de novae, est aujourd'hui comprise comme le résultat de l'explosion d'étoiles en fin de vie), et Galilée, à travers sa lunette, observa les satellites de Jupiter, les phases de Vénus, les montagnes de la Lune et les taches solaires: les astres n'avaient donc pas l'absolue régularité dont on les créditait et, scrutés attentivement, trahissaient de troublantes ressemblances avec la Terre. Réciproquement le mouvement de chute des corps sur la Terre, que la tradition opposait aux mouvements réguliers des astres dans le ciel, se révélait mathématisable. Galilée en conclut qu'il n'y avait pas deux natures, mais une seule, et une seule physique valable partout. Il faudra attendre Newton et la loi de gravitation universelle pour que cette affirmation acquière un contenu véritable. Mais la découverte des lois gravitationnelles, dont relèvent aussi bien la chute de la pomme sur la Terre que le mouvement d'une planète dans le ciel, nécessitait que les deux phénomènes, le terrestre et le céleste, pussent être conçus ensemble, autrement dit

\footnotetext{
${ }^{5}$ Alexandre Koyré fait remarquer que dans le débat sur la finitude ou l'infinité de l'univers, Galilée n'a pas pris parti, le monde restant pour lui plutôt « indéfini » (voir le chapitre « Choses que personne n'a jamais vues », in Du monde clos à l'univers infini [1957], trad. Raissa Tarr, Paris Gallimard, coll. Tel, p. 122 sq.). Cependant, ajoute Koyré, bien que Galilée n'ait jamais pris le parti de rendre le monde infini, cette décision était «impliquée dans la géométrisation de l'espace dont il fut l'un des principaux promoteurs » (p. 126).
} 
que la nature fût déjà, en pensée, unifiée. Le vecteur de cette unification était la géométrie ${ }^{6}-$ non plus langue des astres éternels, mais de l'univers tout entier, ainsi homogénéisé. Tel est le fond sur lequel se déploie le raisonnement de Pascal.

Mais avec Galilée, les choses ne s'arrêtent pas là. De lui, on connaît davantage son Dialogue sur les deux grands systèmes du monde, qui lui valut son second et célèbre procès, en 1633, que son œuvre ultime, ses Discours concernant deux sciences nouvelles, publiés à Leyde en 1638. Pourtant, l'objet principal du Dialogue sur les deux grands systèmes était de prouver que le système de Copernic n'était pas seulement une hypothèse commode pour effectuer des calculs astronomiques, mais une vérité physique, attestée par le phénomène des marées qui en aurait été une conséquence ${ }^{7}$. Le Dialogue expose un grand nombre d'arguments intéressants. Il n'en reste pas moins que la théorie des marées de Galilée, faute de pouvoir prendre en compte l'attraction gravitationnelle, et omettant l'influence majeure de la Lune, est une théorie erronée: il faudra attendre plus d'un demi-siècle pour que Newton donne la première explication à peu près correcte du phénomène. Les Discours, quant à eux, sont tout à fait novateurs, en particulier en ce qui concerne leur première partie consacrée à l'étude de la résistance des matériaux. Le texte s'ouvre sur une visite des arsenaux de Venise. Autour d'une galéasse en attente de lancement, est dressé un appareil de soutènement, bien plus considérable, en proportion, que celui qui entoure les navires plus petits. Un vieil homme assure qu'on procède ainsi avec la galéasse «pour éviter qu'elle se rompe, écrasée par le poids de son énorme masse, alors que les bâtiments plus petits sont exempts de cet inconvénient $^{8} »$. Sagredo, le personnage intelligent et sans a priori des dialogues de Galilée,

\footnotetext{
${ }^{6}$ Galilée a montré que si l'on néglige la résistance de l'air, la trajectoire d'un projectile à la surface de terre est une parabole, et Kepler compris que les orbites des planètes sont des ellipses. Dans les deux cas on a affaire à des coniques (figures obtenues par l'intersection d'un cône et d'un plan), et il fallait cette parenté mathématique pour que Newton pût imaginer une loi de gravitation universelle.

${ }^{7}$ À la fin du siècle, Leibniz chercha à clore le débat entre héliocentrisme et géocentrisme en faisant du mouvement une notion purement relative : le choix du point fixe était donc arbitraire. Mais le mouvement ne se réduit pas à la cinématique, il a aussi des effets dynamiques : les partisans de Newton firent remarquer que lorsqu'on fait tournoyer autour de soi à l'horizontale, au moyen d'une corde, un seau rempli d'eau, la force centrifuge plaque l'eau au fond du seau, alors que si l'on court autour du seau qui, lui, ne fait plus que tourner sur lui-même à l'horizontale, l'eau s'écoule; il faut donc, en physique galiléo-newtonienne, distinguer mouvement relatif et mouvement absolu. C'est précisément ce que Galilée entendait faire, en concevant les marées comme un effet dynamique de la rotation de la terre autour du soleil. (Il a fallu la relativité générale pour que, moyennant les formules adéquates de changement de référentiels d'espace-temps, soit abolie la distinction entre mouvement relatif et mouvement absolu ; dans ce cadre, on peut aussi bien dire que la Terre tourne autour du Soleil que l'inverse.)

${ }^{8}$ Discours concernant deux sciences nouvelles [Discorsi e dimostrazioni matematiche intorno a due nuove scienze, 1638], trad. Maurice Clavelin, Paris, P.U.F., coll. Épiméthée, 1995, p. 7 (Le Opere, éd. G.Barbera, 20 vol., Florence, 1890-1909, t. VIII, p. 50).
} 
critique cette opinion populaire qui veut que beaucoup de projets viables à une petite échelle ne puissent réussir à une échelle plus grande; alors que, estime-t-il, les démonstrations mécaniques reposant sur la géométrie, et les propriétés géométriques d'une figure étant les mêmes à n'importe quelle échelle, une grande machine doit fonctionner de la même manière et aussi bien qu'une petite si les proportions entre les différents éléments qui les composent sont identiques. Salviati, le porte-parole de Galilée, s'accorde avec Sagredo pour critiquer l'opinion populaire. En effet, remarque-t-il, il arrive que l'inverse soit vrai : à savoir que ce qui fonctionne à une grande échelle soit inopérant à une échelle plus petite. Mais il contredit aussi son interlocuteur : «Ne croyez plus, et peut-être avec beaucoup de ceux qui ont étudié la mécanique, que des machines et des constructions faites des mêmes matériaux, reproduisant scrupuleusement les mêmes proportions entre leurs parties, doivent être également ou, pour mieux dire, proportionnellement aptes à résister ou à céder aux assauts et aux chocs venus de l'extérieur, car on peut démontrer géométriquement que les plus grandes sont toujours proportionnellement moins résistantes que les plus petites; de sorte qu'en fin de compte toutes les machines et constructions, qu'elles soient artificielles ou naturelles, ont une limite nécessaire et prescrite que ni l'art ni la nature ne peuvent dépasser, - étant bien sûr entendu que les proportions et les matériaux demeurent toujours identiques ${ }^{9}$. »

Ce que Galilée affirme, c'est que le monde ne saurait être invariant par changement d'échelle $^{10}$ : en termes modernes, on parlerait de non-linéarité. Pensons, pour prendre un exemple très simple, aux géants qui apparaissent dans certains contes, dix fois plus grands qu'un homme ordinaire. Si toutes leurs dimensions sont décuplées, leur volume et leur poids sont multipliés par mille (un homme de 2 mètres et de 100 kilos devient un géant de 20 mètres et de 100000 kilos). Il en résulte que les efforts qui s'exercent par unité de surface de la section d'un os comme le fémur, lorsque le géant est debout, sont multipliés par dix, et qu'au premier pas celui-ci se casse la jambe. De manière générale, par de nombreux arguments et calculs, Galilée fait ressortir «l'impossibilité, non seulement pour l'art, mais pour la nature elle-même, d'accroître leurs constructions jusqu'à des dimensions énormes; ainsi sera-t-il impossible de fabriquer des vaisseaux, des palais ou des temples d'une extrême grandeur, et dont les rames, les vergues, les poutres, les clefs, et en un mot toutes les parties tiendraient encore ensemble; de son côté, la nature sera incapable de produire des arbres d'une taille excessive, puisque les branches, pliant sous leur propre poids, finiraient par se rompre; de la même manière d'ailleurs il sera impossible, aussi bien en ce qui concerne les hommes que les

\footnotetext{
${ }^{9} I d$., p. 8 ( $O p$. VIII, 58). Là où Sagredo entend déduire de la géométrie que toutes les échelles sont équivalentes, Galilée rétorque que c'est géométriquement que l'on peut prouver qu'il n'en est rien.

${ }^{10}$ Les objets fractals, qui offrent la même physionomie à quelque échelle qu'on les observe, semblent faire exception; mais, de fait, ils sont en mathématiques des objets exceptionnels et, dans le monde physique, la similarité de physionomie entre différentes échelles d'observation, quand elle se rencontre, demeure toujours limitée à quelques ordres de grandeur.
} 
chevaux ou les autres animaux, de fabriquer des squelettes capables de durer et remplir régulièrement leurs fonctions, en même temps que ces animaux croîtraient immensément en hauteur : à moins bien entendu d'utiliser une matière beaucoup plus dure et résistante que la matière habituelle, et de déformer leurs os en les agrandissant démesurément, ce qui aboutirait à les rendre monstrueux par la forme et par l'aspect. Pour illustrer brièvement ce que je dis, j'ai représenté un os dont la longueur a été augmentée trois fois seulement, mais dont l'épaisseur a été accrue de telle façon qu'il puisse remplir pour un animal de grande taille la même fonction que le plus petit pour un petit animal; voici les figures sur lesquelles vous remarquez comment l'os agrandi acquiert une forme disproportionnée. Il apparaît donc clairement que, - si l'on voulait conserver chez un géant particulièrement grand la proportion qu'ont les membres chez un homme ordinaire -, il faudrait ou trouver une matière bien plus dure et plus résistante pour en constituer les os, ou admettre que sa robustesse serait proportionnellement beaucoup plus faible que celle des hommes de taille médiocre; sinon, à augmenter sans mesure sa hauteur, on le verrait plier sous son propre poids et s'écrouler ${ }^{11} \gg$.

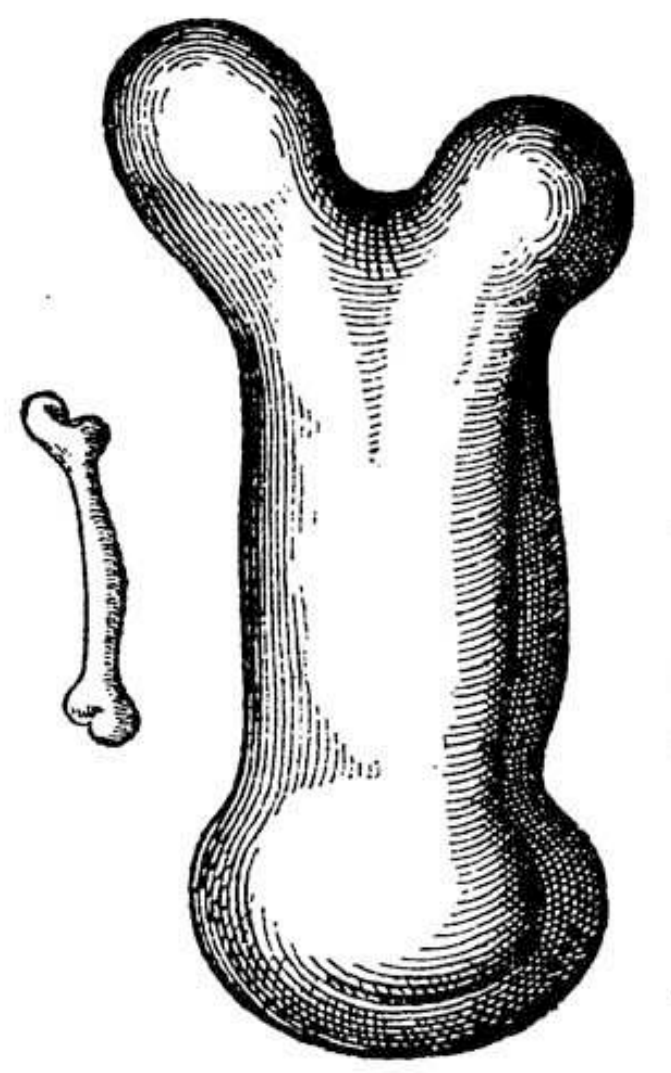

\section{L'importance décisive de l'échelle}

Ces remarques peuvent paraître banales. Il s'en faut pourtant que la pensée moderne en soit pénétrée. Significatif est à cet égard que Sagredo, le Vénitien éclairé, ou Pascal, dans son argument du ciron (qui il est vrai, dans l'argumentation pascalienne, n'a valeur que métaphorique), se montrent sur le sujet plus éloignés de la vérité que le vieillard imbu de sagesse traditionnelle rencontré à l'arsenal. L'affranchissement de la réflexion vis-à-vis des dimensions naturelles offertes à l'expérience apparaît, en quelque sorte, comme une conquête

\footnotetext{
${ }^{11}$ Id., p. 106-107 (Op. VIII, 169-170). Le gros os a trois fois la longueur du petit mais, pour être en mesure de porter une masse $27\left(3^{3}\right)$ fois supérieure, sa section doit également être multipliée par 27, ce qui signifie que son diamètre doit être multiplié par $\sqrt{ } 27=3 \sqrt{ } 3$ (soit à peu près 5,2 ). Le dessin de Galilée exagère un peu le renflement du gros os pour rendre le changement de forme encore plus spectaculaire.
} 
de la modernité. Le biologiste D'Arcy Thompson cite, en exemple, les réflexions de deux grands scientifiques du XIX et du début du XX ${ }^{\mathrm{e}}$ siècle : "Oliver Heaviside avait coutume de dire qu'il n'existe pas d'échelle absolue de taille dans l'Univers, car celui-ci n'a de limite ni dans l'immensément grand ni dans l'immensément petit. C'est l'essence même de la philosophie newtonienne qui devrait nous permettre de généraliser nos concepts et nos déductions d'un extrême à l'autre de l'échelle des grandeurs. Et sir John Herschel affirmait que "celui qui se consacre à l'étude doit renoncer à établir cette distinction entre grand et petit, totalement gommée dans la nature" ${ }^{\prime 2}$. » Même quand une telle position n'est pas ou plus revendiquée, elle a toujours tendance à informer la pensée, empêchant d'accorder aux questions d'échelle l'attention qu'elles méritent. J.B.S. Haldane l'a remarqué en biologie: «Les différences les plus évidentes entre les différents animaux sont des différences de taille mais, pour une raison ou une autre, les zoologistes ont accordé à ces dernières une attention singulièrement réduite. Dans un épais manuel scolaire de zoologie que j’ai sous les yeux, je ne trouve nulle part mentionné que l'aigle est plus grand que le moineau, que l'hippopotame est plus gros que le lièvre - bien que quelques indications soient données du bout des lèvres à propos de la souris et de la baleine. Et pourtant, il serait facile de montrer qu'un lièvre ne saurait avoir les dimensions d'un hippopotame, ni une baleine celles d'un hareng. Pour chaque animal il existe une taille adéquate, et une grande variation de taille entraîne nécessairement un changement de forme ${ }^{13}$. » D'Arcy Thompson, comme après lui Haldane, ont insisté sur le fait que, pour de simples raisons de physique, telles que celles mises au jour par Galilée, la dimension ne saurait être considérée comme un paramètre secondaire dans la caractérisation d'une forme vivante : la taille détermine en effet, dans une large mesure, le type d'organisation possible.

$\mathrm{Si}$ la cellule, unité de structure du vivant, a une taille comprise entre un et cent micromètres (soit du millième au dixième de millimètre), c'est qu'elle ne saurait être trop petite, sous peine de ne pouvoir réunir en son sein les structures moléculaires nécessaires à sa constitution et à son métabolisme, ni trop grosse, sous peine de ne plus être en mesure d'alimenter ce métabolisme. Les besoins en nourriture, en effet, sont proportionnels au volume, tandis que les possibilités d'alimentation sont proportionnelles à la surface. Or, à forme constante, le rapport de la surface au volume varie comme l'inverse de la taille : trop grosse, la cellule ne parvient plus à se nourrir et une division devient nécessaire pour rétablir un rapport surface/volume qui soit viable (sans préjuger des mécanismes effectifs qui

\footnotetext{
${ }_{12}$ D'Arcy Thompson, Forme et Croissance [On Growth and Form, 1917, 1942], éd. John Tyler Bonner [Cambridge University Press, 1961, 1992], trad. Dominique Teyssié, Seuil, coll. Sources du savoir, 1994, p. 4445 .

${ }^{13}$ «On Being the Right Size», Harper's Magazine, mars 1926, repris dans Possible Worlds and Other Essays, Londres, Harper and Brothers, 1927. Une traduction française de ce texte a été publiée dans la revue Conférence, n³3, automne 2011, p. 441-454.
} 
commandent cette division). Les organismes pluricellulaires sont confrontés au même type de problème et, d'une manière générale, «le maintien d'un équilibre adéquat entre la surface et le volume au cours de la croissance est l'un des défis qu'a dû relever l'évolution ${ }^{14}{ }^{»}$, les solutions valables à une certaine échelle trouvant vite leurs limites. La taille croissante pose des difficultés qui réclament des structures de plus en plus complexes pour être surmontées, et « les animaux les plus évolués ne sont pas de plus grande taille que les moins évolués parce qu'ils sont plus complexes, ils sont plus complexes parce qu'ils sont de plus grande taille ${ }^{15} »$. Ainsi, l'oxygénation des insectes par simple diffusion de l'air au long des minces tubes en cul-de-sac que sont les trachées et trachéoles n'est-elle praticable que sur de très courtes distances, d'où s'ensuit que l'épaisseur d'un corps d'insecte ou d'arachnide ne peut guère dépasser un demi-centimètre et que, dans la réalité, les araignées géantes des films d'épouvante, outre qu'elles n'auraient jamais pu atteindre une telle taille, mourraient séance tenante d'asphyxie. Les crustacés, dont le plan anatomique ressemble à celui des insectes, peuvent atteindre de plus grandes dimensions parce qu'ils sont pourvus d'un système respiratoire ; ce sont alors les contraintes mécaniques liées au squelette externe qui viennent limiter leur taille, qui ne dépasse pas celle des crabes et des homards.

Il est à noter que si la taille réclame une organisation plus complexe, elle procure en retour certains avantages : le nombre de prédateurs potentiels diminue, et la régulation de la température, pour les organismes homéothermes, se trouve facilitée par la diminution du rapport entre la surface externe, par laquelle s'opèrent les pertes de chaleur, et le volume dont il faut conserver la chaleur, tandis qu'elle est un défi permanent pour les petits animaux. «En réalité, un animal à sang chaud bien plus petit que la souris est inconcevable ; il ne pourrait ni se procurer ni digérer la quantité de nourriture nécessaire au maintien de sa température à un niveau constant, et c'est pourquoi il n'existe aucun mammifère ni aucun oiseau aussi petit que le plus petit des poissons ou grenouilles. Le caractère désavantageux d'une petite taille est d'autant plus marqué que s'intensifient les pertes de chaleur par conduction, comme dans l'Arctique, ou par convection, comme dans l'océan ${ }^{16}$. »

L'importance de l'échelle sur les phénomènes physiques, bien saisie par Galilée, suffit à comprendre que la forme et l'organisation des êtres vivants soit nécessairement très différente selon l'ordre de grandeur de leur taille - qu'on se place à l'échelle du bacille, de l'insecte ou de l'homme. Les forces de contact, en particulier, n'agissent que proportionnellement à la surface qui leur est offerte, tandis que la force de gravité s'exerce uniformément sur toutes les particules du corps - les premières sont en raison de l'interface intérieur/extérieur, la

\footnotetext{
${ }^{14}$ D'Arcy Thompson, op. cit., p. 60. Haldane le dit également : «L'anatomie comparée est pour une large part l'histoire du combat pour accroître la surface relativement au volume » (art.cit.).

${ }^{15}$ J.B.S. Haldane, art. cit.

${ }^{16}$ D'Arcy Thompson, op. cit., p. 52.
} 
seconde en raison de la masse et donc, à un facteur près, du volume. Du fait que le rapport surface / volume décroît rapidement avec la taille, il résulte que «si la forme des organismes est effectivement dictée par les forces physiques, les créatures de petite taille seront façonnées par des forces de tension superficielle (forces de surface), alors que la forme des organismes plus grands sera régie par des forces gravitationnelles (forces de volume) ». De là le fait que «le phénomène de la forme est de nature essentiellement différent chez les animaux de grande taille et chez ceux de petite taille ». À l'échelle humaine, la force de gravitation est si déterminante que «si [elle] venait à doubler, notre bipédie serait totalement inadaptée, et la plupart des animaux terrestres prendraient la forme de sauriens courts sur pattes ou encore de serpents. [...] En revanche, si l'intensité de la force de gravité diminuait de moitié, nous serions plus légers, plus sveltes et plus actifs, nous aurions un besoin d'énergie, de chaleur et de sang moins important, et notre cœur et nos poumons pourraient être plus petits ${ }^{17}{ }$. Même s'il n'y a pas de seuil absolu de viabilité pour tel ou tel type de forme et d'organisation, il n'en existe pas moins des discontinuités: "L'échelle de taille des mammifères s'interrompt brutalement, vers le bas, à un poids d'environ 5 grammes, celle des coléoptères à une longueur d'environ un demi-millimètre, et chaque groupe d'animaux a ses propres limitations de taille, vers le haut et vers le bas ${ }^{18}$. $\gg$ Comme le souligne encore Haldane, pour chaque type d'animal il existe une taille optimale. Il n'y a que dans l'imagination de Swift et de ses lecteurs que Gulliver, les Brobdingnags et les Lilliputiens peuvent coexister : dans la réalité, une même forme organique ne saurait être viable à des échelles aussi différentes.

\footnotetext{
${ }^{17} I d$., p. 9-10 (préface de Stephen Jay Gould), p. 61 et p. 57. Plus la taille d'un organisme croît, plus la pesanteur devient pour lui une contrainte importante, et un danger - alors qu'elle exerce peu d'effet sur les organismes de petites dimensions. Galilée l'avait remarqué : "Qui ne voit qu'un cheval se rompra les os, s'il tombe d'une hauteur de trois ou quatre coudées [une coudée vaut environ 45 centimètres], mais qu'un chien, dans les mêmes conditions, ou un chat tombant d'une hauteur de huit ou dix coudées, ne se feront aucun mal, pas plus qu'un grillon lâché d'une tour ou une fourmi se précipitant depuis l'orbe lunaire ?» (Discours..., op. cit., p. 9). Cela, parce que la résistance que l'air oppose à la chute d'un objet est proportionnelle à la surface de cet objet, tandis que la force de gravitation est proportionnelle à sa masse. En revanche, les forces de tension superficielle, qui n'ont rien pour nous effrayer, sont très dangereuses pour les insectes. "Un homme qui sort d'un bain porte sur lui un film d'eau épais d'environ un demi-millimètre, pesant au total à peu près une livre. Une souris trempée doit porter l'équivalent de son propre poids en eau. Une mouche trempée, elle, a à soulever plusieurs fois son propre poids et, comme chacun sait, une mouche qui a été mouillée par de l'eau ou un autre liquide se trouve en très mauvaise posture. Un insecte qui cherche à boire court un danger aussi grand qu'un homme qui, dans sa quête de nourriture, se penche au-dessus d'un précipice. Si jamais l'insecte tombe sous l'emprise de la tension superficielle de l'eau - c'est-à-dire, se retrouve mouillé - il est probable qu'il restera tel jusqu'à mourir d'asphyxie. Quelques insectes, comme les coléoptères dulçaquicoles, ont trouvé le moyen d'échapper à la mouillure ; mais la majorité d'entre eux doivent se tenir à distance respectable du liquide qu'ils absorbent au moyen d'une longue trompe » (Haldane, art. cit.).

${ }^{18}$ D'Arcy Thompson, op. cit., p. 65.
} 


\section{Les raisons d'une ignorance}

Il est remarquable que Galilée, en même temps qu'il travaillait avec constance à démanteler le cosmos traditionnel, et semblait du même coup ouvrir la voie à une conception purement relative de la mesure, ait aussi à la fin de sa vie donné les moyens de refonder l'idée de mesure absolue sur de nouvelles bases, en montrant que le monde, même considéré more mathematico, ne saurait être invariant par changement d'échelle ${ }^{19}$. L'opposition ou la disjonction entre quantitatif et qualitatif s'en trouve largement affaiblie, puisque la quantité détermine dans une large mesure les qualités possibles, et que les qualités ne peuvent se réaliser qu'à l'intérieur de limites quantitatives assez peu extensibles.

Il est non moins remarquable de constater à quel point la pensée moderne semble éluder ce rapport. Pour Aristote, le seul mouvement perpétuel concevable était la description périodique d'une trajectoire fermée, parce que la nature n'en donnait pas d'autre exemple. Il fallut s'abstraire du monde tel qu'il se donne à nos sens pour pouvoir formuler le principe d'inertie selon lequel, en l'absence de toute force extérieure, un corps conserve sa vitesse en intensité et en direction (dans un référentiel dit galiléen). Mais c'est comme si cette abstraction, fort utile en l'occurrence, avait ensuite présidé à l'appréhension du monde en général, envisagé comme une accumulation de corps primitivement indépendants les uns des autres et entrant ensuite en interaction. En procédant de cette manière - qu'encourageait le développement des conceptions individualistes, qu'elle favorisait en retour -, il est devenu très difficile de saisir le caractère essentiel de la taille, car «l'effet de l'échelle ne dépend pas d'un objet en lui-même, mais de sa relation avec tout l'environnement, le milieu ; cet effet est conforme à la "place de l'objet dans la nature", à son champ d'action et de réaction dans l'Univers. Tout le fonctionnement de la nature est parfaitement ajusté, et la taille de chaque objet est précisément adaptée à ce fonctionnement ${ }^{20} \gg$.

\footnotetext{
${ }^{19}$ Par exemple, dire d'un homme de 2,50 mètres qu'il est un géant peut s'entendre autrement que par seule comparaison avec la taille moyenne observée : la taille de 2,50 mètres pourrait voisiner la limite au-delà de laquelle la forme et l'organisation humaines ne sont plus viables. Un homme de 2,50 mètres serait très grand non relativement à notre expérience empirique, « provinciale » du réel, parce que nous n'avons jamais vu d'hommes de 3 mètres (comme les Anciens pouvaient juger extrêmement rapide un cheval au galop parce qu'ils ne connaissaient pas les trains à grande vitesse et les avions), un homme de 2,50 mètres serait très grand dans l'absolu, parce qu'un homme de 3 mètres est impossible.

${ }^{20}$ D'Arcy Thompson, op. cit., p. 45. Alexandre Koyré note qu' «en adoptant universellement le modèle atomique d'analyse et en l'appliquant à des événements et des actions donnés globalement, on fait apparaitre ces totalités non pas comme réelles mais comme n'étant que des résultats mathématiques et des additions de facteurs élémentaires sous-jacents. Ce type d'analyse conduisit à la mésinterprétation nominaliste de la relation entre un totum et ses parties; mésinterprétation qui, en fait, aboutit à nier complètement les totalités (un totum réduit à la pure addition de ses parties n'est pas un totum), ce que la pensée des $\mathrm{XIX}^{\mathrm{e}}$ et $\mathrm{XX}^{\mathrm{e}}$ siècles eut tant de peine à
} 
Une autre raison à la sous-estimation, pour ainsi dire systématique, de l'importance des questions de grandeur dans la définition même des choses tient à la progressive autonomisation, au cours de la période moderne, de ce qu'on appelait d'abord philosophie naturelle, aboutissant au XIX ${ }^{\mathrm{e}}$ siècle à une séparation quasi totale entre sciences et lettres ${ }^{21}$. Selon Heidegger, l'université aurait dû être organisée en trois grandes facultés : sciences, lettres, et une troisième entité qui aurait regroupé mathématiques et philosophie. Les choses ne se sont pas passées ainsi : les mathématiques ont été versées du côté des sciences, en considération de leur implication essentielle dans la physique et, plus généralement, dans l'idéal scientifique modernes, la philosophie a été versée du côté des lettres, en vertu d'un lien rémanent avec les humanités, et parce qu'elle se trouvait dépossédée par la nouvelle science de ses anciennes prétentions scientifiques. Dès lors, la pensée philosophique va avoir tendance à faire de nécessité vertu, à afficher une indifférence, voire un dédain de plus en plus marqués à l'égard des questions quantitatives, abandonnées aux scientifiques et à leurs calculs - allant souvent, semble-t-il, jusqu'à mettre un point d'honneur à ignorer le nombre au moment même où celui-ci prenait de plus en plus d'importance dans la conduite des affaires humaines.

Prenons l'exemple de la philosophie politique. En Grèce ancienne on était très conscient du rôle déterminant joué par la taille d'une population. C'est pourquoi Platon, dans ses Lois $(\mathrm{V}, 737 \mathrm{e}-738 \mathrm{~b})$ se souciait du nombre de citoyens propriétaires de sa cité idéale (fixé à $7 !=5040$, pour des raisons de divisibilité par tous les nombres jusqu'à dix - mais c'est l'ordre de grandeur qui nous intéresse ici). Aristote, dans sa Politique, se montrait moins précis, mais il n'en accordait pas moins une grande attention à la question : "Ce qui résulte clairement aussi de l'examen des faits, c'est qu'il est difficile et peut-être impossible pour un État dont la population est trop nombreuse, d'être régi par de bonnes lois. En tout cas, parmi les États ayant la réputation d'être sagement gouvernés, nous n'en voyons aucun se relâcher de sa vigilance en ce qui touche le chiffre de sa population. Ce point est encore rendu évident sur la foi d'arguments théoriques. La loi est, en effet, un certain ordre ( $\tau \alpha \dot{\xi} \xi \varsigma$ ), et la bonne

\footnotetext{
surmonter » («Sens et portée de la synthèse newtonienne » [1948], in Études newtoniennes, Paris, Gallimard, coll. Bibl. des idées, 1968, p.42).

${ }^{21}$ À la fin du XVII ${ }^{\mathrm{e}}$ siècle on ne concevait pas encore cette séparation. En témoigne le dictionnaire de Furetière [1690] :

LETTRES : se dit aussi des sciences. C'est un homme de Lettres, il a été élevé dans les Lettres, il a des Lettres. Les Barbares sont ennemis des Lettres, ne les cultivent point. [...] On appelle les Lettres humaines, \& abusivement les belles Lettres, la connaissance des poètes \& des orateurs; au lieu que les vraies belles Lettres sont la Physique, la Géométrie, \& les sciences solides.

SCIENCE : Connaissance des choses, acquise par une grande lecture, ou une longue méditation. Érasme avait un grand fonds de science, de doctrine. [...] La Philosophie comprend toutes les sciences. [...] On appelle les sciences humaines, la connaissance des Langues, de la Grammaire, de la Poésie, de la Rhétorique, \& autres choses qu'on apprend dans les Humanités.
} 
législation est nécessairement un bon ordre ; or une population qui atteint un chiffre trop élevé ne peut pas se prêter à un ordre ; y introduire de l'ordre serait dès lors un travail relevant d'une puissance divine, celle-là même qui assure la cohésion des diverses parties de notre Univers $(\tau o ̀ ~ \pi \bar{\alpha} v)$. [...] Il existe en fait une certaine mesure de grandeur pour un État, comme il y en a aussi pour tout le reste, animaux, plantes, instruments : aucun de ces êtres, s'il est trop petit ou d'une grandeur excessive, ne conservera sa capacité à remplir sa fonction, mais tantôt il aura entièrement perdu sa nature, et tantôt verra sa condition viciée $e^{22}$. On voit que pour Aristote, la question de la taille était fondamentale, puisque c'était seulement si cette taille se maintenait entre des bornes assez étroites qu'il s'avérait possible de donner à la cité de bonnes lois - aussi différentes ces lois pussent-elles être d'une cité à une autre. La taille n'était pas un paramètre qui venait s'ajouter après coup au système des lois, elle était le préalable qui permettait, ou non, à un système de lois de remplir son rôle. Si l'on effectue un grand bond jusqu'au XVIII ${ }^{\mathrm{e}}$ siècle, on s'aperçoit que les philosophes des Lumières se livraient volontiers à l'examen des différents types de régimes in abstracto. Ils ne manquaient pourtant pas, aussitôt après, de se souvenir de l'importance des dimensions du territoire et de la population, et tenaient compte de ces données pour déterminer ce qui, selon eux, convenait le mieux à tel ou tel type de situation. Ainsi, Montesquieu ou Rousseau s'accordaient-ils à considérer qu'un régime démocratique n'était viable que pour de petites populations («un État très petit, où le peuple soit facile à rassembler, et où chaque citoyen puisse aisément connaître tous les autres » est le premier requisit énoncé dans Le Contrat social). À partir du $\mathrm{XIX}^{\mathrm{e}}$ siècle cependant, on a l'impression qu'il n'y eut plus guère que les utopistes et les expérimentateurs socialistes pour prendre vraiment au sérieux les questions de nombre, pour en faire un élément déterminant de l'organisation qu'ils imaginaient ou cherchaient à mettre en œuvre (Charles Fourier avec ses phalanstères accueillant environ 1600 membres, Robert Owen avec ses parallélogrammes réunissant de 500 à 3000 personnes, Horace Greeley avec ses associations qui devaient compter de quelques centaines à quelques milliers de membres). Aujourd'hui, l'emploi du même terme de « démocratie » pour évoquer le système politique de l'Athènes $d u V^{e}$ siècle avant notre ère comme celui de l'Inde contemporaine ne semble gêner personne, comme s'il y avait une essence de la démocratie qui se conservait inchangée lorsqu'on passe d'une population de 400000 habitants, dont un dixième seulement de citoyens, à une population de plus de 1,2 milliards d'habitants, avec plus de 700 millions de votants aux élections générales (soit respectivement 3000 et 18000 fois plus dans l'Inde

\footnotetext{
${ }^{22}$ Trad. Jules Tricot, Vrin, coll. Bibl. des Textes philosophiques, 1995, 1326a. Il n'y avait pas que les philosophes pour se soucier de la taille des cités : ces dernières prenaient des mesures concrètes afin de contrôler leur taille, en fondant des colonies à même de les décharger de leur excédent de population.
} 
d'aujourd'hui que dans l'Athènes classique). Leopold $\mathrm{Kohr}^{23}$, au XX $\mathrm{XX}^{\mathrm{e}}$ siècle, fut l'un des rares penseurs à souligner que la première caractéristique d'une société est la taille de sa population, et qu'il y a beaucoup plus de ressemblances, ou beaucoup moins de différences, entre une «démocratie» et une «monarchie» ayant quelques centaines de milliers ou quelques millions de ressortissants qu'entre deux démocraties, ou deux monarchies, l'une ayant cent, mille ou dix mille fois plus d'habitants que l'autre ${ }^{24}$. Appliqués à des échelles aussi différentes, les mots de «démocratie», d' «aristocratie» ou de «monarchie » deviennent des trompe-l'œil, laissant croire à une essence commune là où, du simple fait du nombre, il ne peut à peu près rien subsister de commun.

La question de la taille, du nombre, demeure pour une large part un point aveugle de la réflexion philosophique moderne et contemporaine, qui s'ingénie à définir des concepts en dehors de toute référence quantitative, en oubliant que la plupart des concepts ont été élaborés à l'intérieur d'un certain horizon au-delà duquel ils perdent leur sens, ou deviennent en gardant leur nom quelque chose de tout à fait différent - la communauté de terme semant alors la confusion dans la pensée. On est tenté d'attribuer pareil travers à une «perte des

\footnotetext{
${ }^{23}$ Kohr est né en 1909 dans la bourgade d'Oberndorf, près de Salzbourg, et s'est éteint à Gloucester, au pays de Galles, en 1994. Quoique l'Anschluss l'ait conduit à s'expatrier aux États-Unis (il a enseigné à l'Université de Rutgers, puis à l'Université de Porto Rico), et à devenir citoyen américain, toute sa vie il a gardé la conviction que l'unité de distance pertinente pour organiser une société saine était de l'ordre de celle séparant Oberndorf de Salzbourg, la capitale du Land, située à vingt-deux kilomètres. Par ailleurs, Kohr estimait qu'aucune société humaine, quelle que fût sa forme d'organisation, ne pouvait vivre correctement au-delà d'un maximum absolu de douze à quinze millions de membres. Il a été un des premiers à recevoir le prix Nobel alternatif, en 1983. Son ouvrage le plus important est The Breakdown of Nations [1957] (rééd. Totnes (Devon), Green Books, 2001).

${ }^{24} \mathrm{Au}$ titre des quelques exceptions à la négligence générale envers le caractère essentiel du nombre, citons Georg Simmel, auteur d'une étude intitulée «The Number of Members as Determining the Sociological Form of the Group », publiée dans l'American Journal of Sociology, n 8, 1902, p. 1-46 et 158-196 (trad. de l'allemand par A.W. Small). Dans ce long article Simmel fait remarquer, entre autres, que si un authentique régime démocratique réclame une population limitée, un régime aristocratique ne saurait non plus s'accommoder d'un trop grand nombre de ressortissants; l'aristocratie doit en effet compter davantage de membres pour être en mesure de contrôler une population qui s'accroît, mais rester suffisamment restreinte pour que sa cohésion soit entretenue par une connaissance directe entre membres et des alliances matrimoniales parcourant tout le corps. Il existe donc une limite absolue à la taille d'une société aristocratique. Simmel souligne aussi le fait que plus une société est nombreuse, plus la division du travail doit y être poussée, parce que seule cette dernière est à même de maintenir une certaine unité au sein d'une population qui, sans cela, se fragmenterait à la première occasion (dans les termes de Durkheim, la « solidarité organique », fondée sur l'interdépendance des individus spécialisés chacun dans une tâche, se substitue à la « solidarité mécanique », de proximité, qui lie les individus au sein des petites communautés) ; autrement dit, une extrême division du travail n'est pas seulement permise par une société nombreuse, elle est aussi une condition d'existence de cette société en tant que telle - d'une manière qui n'est pas sans rappeler la complexité nécessairement croissante des organismes lorsque leur taille augmente, pointée par Haldane.
} 
sens », à tout le moins à une perte de la confiance dans les témoignages des sens et à une diminution de l'attention qu'on leur porte, qui seraient caractéristiques de la modernité. Le corps humain cesse d'être un étalon de mesure pour le monde (comme l'illustre l'effacement par le système métrique d'unités comme le pouce, le pied, la coudée, la toise etc.) et, du même mouvement, se perd concrètement la notion de dimension absolue. Cependant, si Galilée a confessé son admiration pour Aristarque et Copernic, qui ont su imaginer le système héliocentrique parce que chez eux «la raison a pu faire une telle violence aux sens jusqu'à devenir, malgré les sens, maîtresse de leurs croyances ${ }^{25}$ », on a vu qu'il a également remarqué et souligné que le monde n'est pas invariant par changement d'échelle. Il en résulte que le nœud entre quantité et qualité est impossible à défaire. Effacé sous son ancienne guise cosmique par l'appréhension mathématique du livre de la nature, il réapparaît aussitôt, précisément, sous forme mathématique. Les non-linéarités (autrement dit, les variations d'une grandeur par rapport à une autre qui ne sont pas de simple proportionnalité) font que le monde n'est pas invariant par homothétie, et ne saurait l'être. Contribue à nous masquer ce fait l'inflation contemporaine des images et du « virtuel ». Les images nous habituent à considérer les choses ou les êtres représentés indépendamment de l'échelle à laquelle ces choses ou ces êtres existent. Sur un écran d'ordinateur, nous pouvons même faire varier la taille à notre guise : non seulement la mesure est relative, mais elle est à notre disposition. Dans la réalité, il en va autrement. Lorsque la taille croît (ou décroît), la forme ne saurait se maintenir longtemps: les non-linéarités engendrent des seuils, où un système bascule d'un type de comportement à un autre, des «catastrophes» qui, dans certains cas, peuvent être éminemment bénéfiques et souhaitables et, dans d'autres, sont synonymes de destruction, d'effondrement (comme le sens courant pris par le terme en français l'indique assez). Les non-linéarités donnent un sens à la notion de mesure absolue. Quitte à comprendre que ce caractère absolu n'est pas un nombre précis, mais un ordre de grandeur qui, pour tout objet, fait partie de son essence. En insistant sur ce point, la science moderne pourrait contribuer à refonder une forme de sagesse cosmique, plutôt que de la faire oublier.

\footnotetext{
${ }^{25}$ Dialogue sur les deux grands systèmes du monde, trad. René Fréreux et François de Gandt, Seuil, 1992, p. 331 et 337 (Op. VII, 355, 362-363).
} 